\title{
The Visualization of the Space Probability Distribution for a Moving Particle: In a Single Ring-Shaped Coulomb Potential
}

\author{
Yuan You, ${ }^{1}$ Fa-Lin Lu, ${ }^{1}$ Dong-Sheng Sun, ${ }^{1}$ Chang-Yuan Chen, ${ }^{1}$ and Shi-Hai Dong ${ }^{2}$ \\ ${ }^{1}$ New Energy and Electronic Engineering, Yancheng Teachers University, Yancheng 224002, China \\ ${ }^{2}$ Laboratorio de Información Cuántica, CIDETEC, Instituto Politécnico Nacional, Unidad Profesional Adolfo López Mateos, \\ 07700 Ciudad de México, Mexico
}

Correspondence should be addressed to Yuan You; yuanyou_w@163.com, Chang-Yuan Chen; yctcccy@163.net, and Shi-Hai Dong; dongsh2@yahoo.com

Received 4 March 2017; Accepted 9 April 2017; Published 8 October 2017

Academic Editor: Saber Zarrinkamar

Copyright (C) 2017 Yuan You et al. This is an open access article distributed under the Creative Commons Attribution License, which permits unrestricted use, distribution, and reproduction in any medium, provided the original work is properly cited. The publication of this article was funded by SCOAP $^{3}$.

\begin{abstract}
We first present the exact solutions of the single ring-shaped Coulomb potential and then realize the visualizations of the space probability distribution for a moving particle within the framework of this potential. We illustrate the two-dimensional (contour) and three-dimensional (isosurface) visualizations for those specifically given quantum numbers $(n, l, m)$ essentially related to those so-called quasi-quantum numbers $\left(n^{\prime}, l^{\prime}, m^{\prime}\right)$ through changing the single ring-shaped Coulomb potential parameter $b$. We find that the space probability distributions (isosurface) of a moving particle for the special case $l=m$ and the usual case $l \neq m$ are spherical and circularly ring-shaped, respectively, by considering all variables $\vec{r}=(r, \theta, \varphi)$ in spherical coordinates. We also study the features of the relative probability values $P$ of the space probability distributions. As an illustration, by studying the special case of the quantum numbers $(n, l, m)=(6,5,1)$, we notice that the space probability distribution for a moving particle will move towards the two poles of the $z$-axis as the relative probability value $P$ increases. Moreover, we discuss the series expansion of the deformed spherical harmonics through the orthogonal and complete spherical harmonics and find that the principal component decreases gradually and other components will increase as the potential parameter $b$ increases.
\end{abstract}

\section{Introduction}

Since the ring-shaped noncentral potentials have potential applications in quantum chemistry and nuclear physics (e.g., they might describe the molecular structure of benzene and interaction between the deformed nucleuses), it is not surprising that their relevant investigations have attracted much attention [1-20]. Based on a previous study, we have known that this type of ring-shaped noncentral potential can be solved in spherical coordinates and also the Hamiltonian system with the hidden symmetry makes the bound state energy levels possess an "accidental" degeneracy, which arises from the SU(2) invariance of the Schrödinger Hamiltonian [1]. Generally speaking, the most popular ring-shaped noncentral potentials are identified as the Coulomb or harmonic oscillator plus the ring-shaped part $1 /\left(r^{2} \sin ^{2} \theta\right)$. In this work, we are concerned with only the single ring-shaped Coulomb potential in the limited space.

Many authors have obtained the radial and polar angular differential equations and also got their solutions in recent studies $[7,8,13,14,21]$, but the whole space $\vec{r}=(r, \theta, \varphi)$ probability distributions of the moving particle in the single ring-shaped noncentral fields have never been reported due to the difficult computational skill that is required in the programming. The main contributions mentioned above are concerned either with the radial part in the spherical shell $(r, r+d r)$ or with the angular parts in volume angle $d \Omega[22$, $23]$. This means that these studies are only related to one or two of three variables $(r, \theta, \varphi)$. To illustrate comprehensively the space probability distribution of the moving particle confined in the ring-shaped noncentral Coulomb potential, 
the aim of this work is to realize their two-dimensional (contour) and three-dimensional (isosurface) visualizations by considering all variables. Such studies have never been done to the best of our knowledge.

The rest of this work is organized as follows. In Section 2, we first present the solutions of the studied quantum system and give the concrete expressions of the angular wave functions in order to perform a comparison with the usual spherical harmonics and also show their distinct properties. In Section 3, we make use of the calculation formula of the space probability distribution to illustrate the visualizations of various cases for different choices of the parameters by overcoming the calculation skills in MATLAB program. In Section 4, we discuss the variation of the space probability distribution with the number of radial nodes and the variations with the relative probability value $P$ and those with the negative and positive ring-shaped Coulomb potential parameter $b$. The expansion coefficients of the deformed spherical harmonics are calculated in Section 5. Some concluding remarks are given in Section 6.

\section{Exact Solutions to Single Ring-Shaped Coulomb Potential}

The single ring-shaped Coulomb potential is given by

$$
V(r, \theta)=-\frac{Z e^{2}}{r}+\frac{\hbar^{2}}{2 M r^{2}} \frac{b}{\sin ^{2} \theta},
$$

and the Schrödinger equation is written as

$$
\left[-\frac{\hbar^{2}}{2 M} \nabla^{2}-\frac{Z e^{2}}{r}+\frac{\hbar^{2}}{2 M r^{2}} \frac{b}{\sin ^{2} \theta}\right] \Psi(\vec{r})=E \Psi(\vec{r}) .
$$

Take the wave function of the following form:

$$
\Psi(\vec{r})=\frac{1}{\sqrt{2 \pi}} \frac{u(r)}{r} H(\theta) e^{i m \varphi},
$$

where $m=0, \pm 1, \pm 2, \ldots$ Substitute (3) into (2) and get the respective radial and angular differential equations as

$$
\begin{aligned}
& \frac{1}{\sin \theta} \frac{d}{d \theta}\left(\sin \theta \frac{d H(\theta)}{d \theta}\right) \\
& +\left(\lambda-\frac{m^{2}}{\sin ^{2} \theta}-\frac{b}{\sin ^{2} \theta}\right) H(\theta)=0, \\
& \frac{d^{2} u(r)}{d r^{2}}+\left(\frac{2 M E}{\hbar^{2}}+\frac{2 M}{\hbar^{2}} \frac{Z e^{2}}{r}-\frac{\lambda}{r^{2}}\right) u(r)=0 .
\end{aligned}
$$

Take a new variable transform $x=\cos \theta$; hence, (4a) becomes

$$
\begin{aligned}
& \left(1-x^{2}\right) \frac{d^{2} H(x)}{d x^{2}}-2 x \frac{d H(x)}{d x}+\left(\lambda-\frac{m^{2}+b}{1-x^{2}}\right) H(x) \\
& =0 .
\end{aligned}
$$

Its solutions were given by the universal associated Legendre polynomials [22]:

$$
\begin{aligned}
& H_{l^{\prime} m^{\prime}}(x)=N_{l^{\prime} m^{\prime}} P_{l^{\prime}}^{m^{\prime}}(x)=N_{l^{\prime} m^{\prime}}\left(1-x^{2}\right)^{m^{\prime} / 2} \\
& \cdot \sum_{\nu=0}^{\left[\left(l^{\prime}-m^{\prime}\right) / 2\right]} \frac{(-1)^{\nu} \Gamma\left(2 l^{\prime}-2 v+1\right)}{2^{l^{\prime}} \nu !\left(l^{\prime}-m^{\prime}-2 \nu\right) ! \Gamma\left(l^{\prime}-v+1\right)} x^{l^{\prime}-m^{\prime}-2 v} \\
& =\sqrt{\frac{\left(2 l^{\prime}+1\right) \Gamma\left(l^{\prime}+m^{\prime}+1\right)}{2^{2 m^{\prime}+1}\left(l^{\prime}-m^{\prime}\right) !\left[\Gamma\left(m^{\prime}+1\right)\right]^{2}}\left(1-x^{2}\right)^{m^{\prime} / 2}{ }_{2} F_{1}\left[-l^{\prime}\right.} \\
& \left.+m^{\prime}, l^{\prime}+m^{\prime}+1 ; m^{\prime}+1 ; \frac{1-x}{2}\right],
\end{aligned}
$$

where

$$
\begin{aligned}
m^{\prime} & =\sqrt{m^{2}+b}>0, \\
l^{\prime} & =n_{\theta}+m^{\prime}, \\
\lambda & =l^{\prime}\left(l^{\prime}+1\right), \\
n_{\theta} & =0,1,2, \ldots, \\
N_{l^{\prime} m^{\prime}} & =\sqrt{\frac{\left(2 l^{\prime}+1\right)\left(l^{\prime}-m^{\prime}\right) !}{2 \Gamma\left(l^{\prime}+m^{\prime}+1\right)} .}
\end{aligned}
$$

For $m^{\prime}=-\sqrt{m^{2}+b}<0$, notice that the differential equation (5) keeps invariant if $m^{\prime}$ is replaced by $-m^{\prime}$, and thus we know that $P_{l^{\prime}}^{-m^{\prime}}(x)$ is also the solution of (5) and its definition is given by $[24,25]$

$$
P_{l^{\prime}}^{-m^{\prime}}(x)=(-1)^{m} \frac{\Gamma\left(l^{\prime}-m^{\prime}+1\right)}{\Gamma\left(l^{\prime}+m^{\prime}+1\right)} P_{l^{\prime}}^{m^{\prime}}(x) .
$$

On the other hand, in terms of the spherical harmonics $Y_{l m}(\theta, \varphi)[26,27]$,

$$
\begin{aligned}
& Y_{l m}(\theta, \varphi) \\
& \quad=(-1)^{m} \sqrt{\frac{2 l+1}{4 \pi} \frac{\Gamma(l-m+1)}{\Gamma(l+m+1)}} P_{l}^{m}(\cos \theta) e^{i m \varphi} .
\end{aligned}
$$

We can define deformed spherical harmonics $Y_{l^{\prime} m^{\prime}}(\theta, \varphi)$

$$
\begin{aligned}
& Y_{l^{\prime} m^{\prime}}(\theta, \varphi) \\
& \quad=(-1)^{m} \sqrt{\frac{2 l+1}{4 \pi} \frac{\Gamma\left(l-m^{\prime}+1\right)}{\Gamma\left(l+m^{\prime}+1\right)}} P_{l}^{m^{\prime}}(\cos \theta) e^{i m \varphi},
\end{aligned}
$$


TABLE 1: Analytical expressions of $Y_{l^{\prime} m^{\prime}}(\theta, \varphi)$ for special cases $b=0$ and $b \neq 0$.

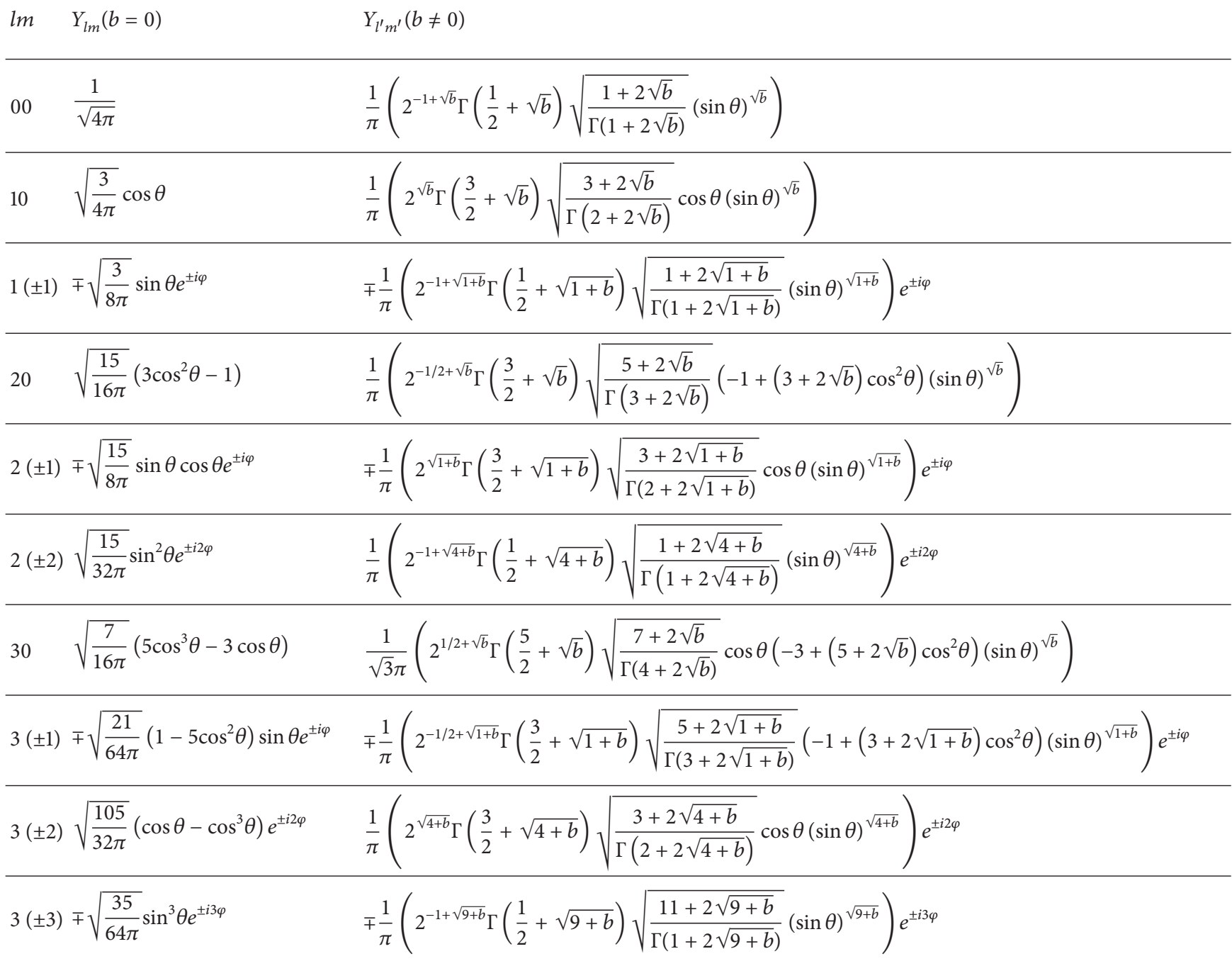

with the following property:

$$
Y_{l^{\prime}\left(-m^{\prime}\right)}(\theta, \varphi)=(-1)^{m} Y_{l^{\prime} m^{\prime}}(\theta, \varphi)^{*} .
$$

Obviously, $m^{\prime}$ is equal to $m$ when $b=0$, and (8), (10), and (11) will reduce to the results in the central field. It is worth pointing out that all states $Y_{l m}(\theta, \varphi)$ are $2 l+1$ for a certain value $l$, but states $Y_{l^{\prime} m^{\prime}}(\theta, \varphi)$ are $2(m \neq 0)$, or $1(m=0)$. This arises from the fact that the ring-shaped potential reduces the symmetry of the system and the degeneracy.

In Table 1, we list the analytical expressions of some given deformed spherical harmonics $Y_{l^{\prime} m^{\prime}}(\theta, \varphi)$ in the special cases of potential parameter $b=0$ and $b \neq 0$.

Now, let us consider the radial differential equation. Substitute $\lambda=l^{\prime}\left(l^{\prime}+1\right)$ into $(4 \mathrm{~b})$ and take $\chi=\tau r, s=$ $2 M Z e^{2} / \hbar^{2}$, and $\tau=\left(M Z e^{2} / \hbar^{2}\right) \sqrt{-\hbar^{2} / 2 M E}$; then, (4b) can be rewritten as

$$
\frac{d^{2} u(\chi)}{d \chi^{2}}+\left(\frac{s}{\chi}-\frac{1}{4}-\frac{l^{\prime}\left(l^{\prime}+1\right)}{\chi^{2}}\right) u(\chi)=0 .
$$

Its solutions are nothing but the Coulomb case [28]; that is,

$$
\begin{aligned}
u_{n^{\prime} l^{\prime}}(r)= & \frac{1}{\Gamma\left(2 l^{\prime}+2\right)}\left[\frac{Z}{a_{0}} \frac{\Gamma\left(n^{\prime}+l^{\prime}+1\right)}{n_{r}^{\prime} !\left(n^{\prime}\right)^{2}}\right]^{1 / 2} \\
& \cdot\left(\frac{2 Z r}{a_{0} n^{\prime}}\right)^{l^{\prime}+1} \\
& \cdot e^{-Z r / a_{0} n^{\prime}} F\left(-n_{r}, 2 l^{\prime}+2, \frac{2 Z r}{a_{0} n^{\prime}}\right)
\end{aligned}
$$

where $n^{\prime}=n_{r}+l^{\prime}+1=n_{r}+n_{\theta}+m^{\prime}+1, n_{r}=0,1,2, \ldots$, and $a_{0}=\hbar^{2} / M e^{2}$ is the Bohr radius. Thus, the wave function in the whole space is written as

$$
\Psi_{n^{\prime} l^{\prime} m}(\vec{r})=\frac{1}{\sqrt{2 \pi}} \frac{u_{n^{\prime} l^{\prime}}(r)}{r} H_{l^{\prime} m^{\prime}}(\cos \theta) e^{i m \varphi}
$$


TABLE 2: Three-dimensional (isosurface) space probability distributions with a section plane.

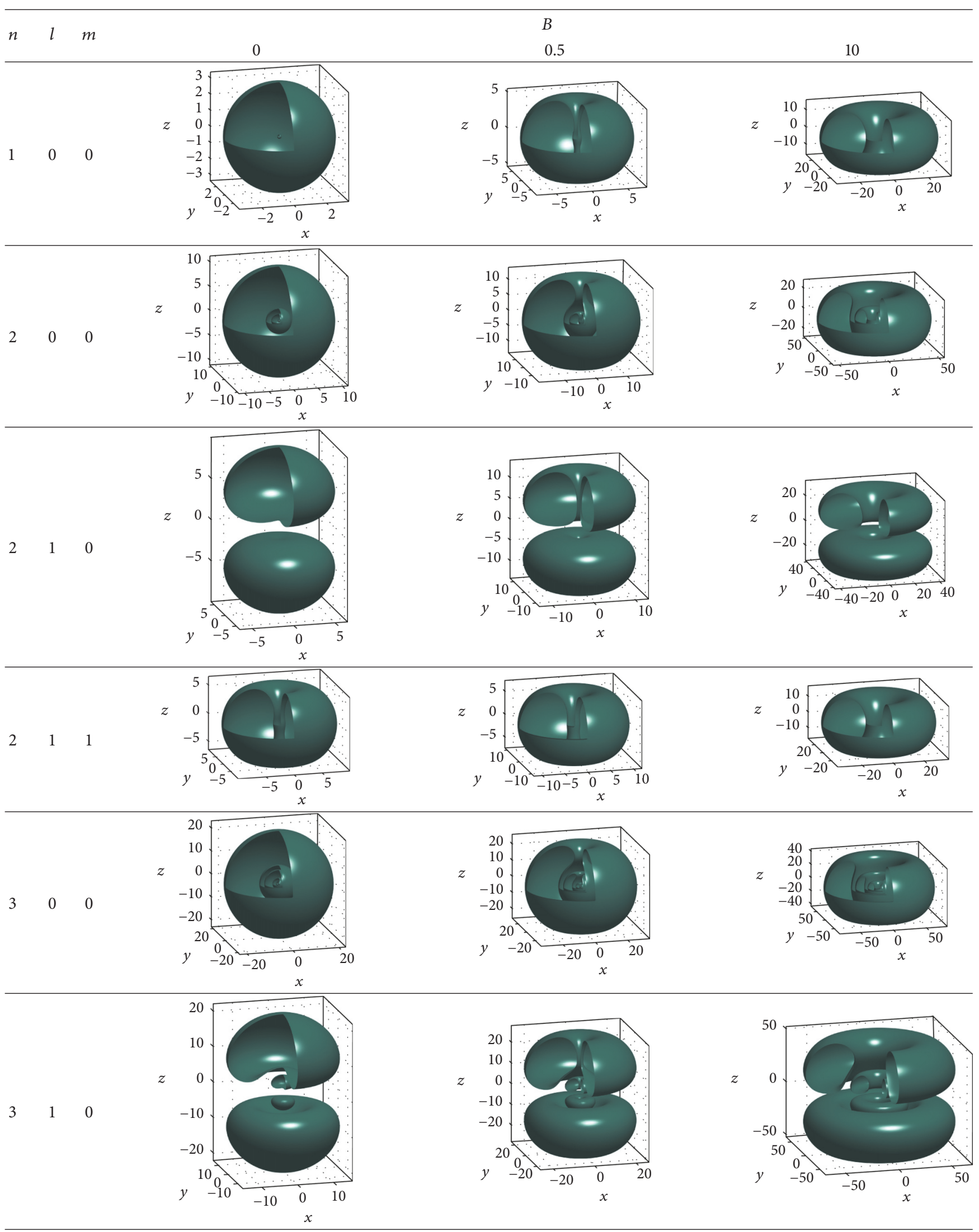


TABLe 2: Continued.

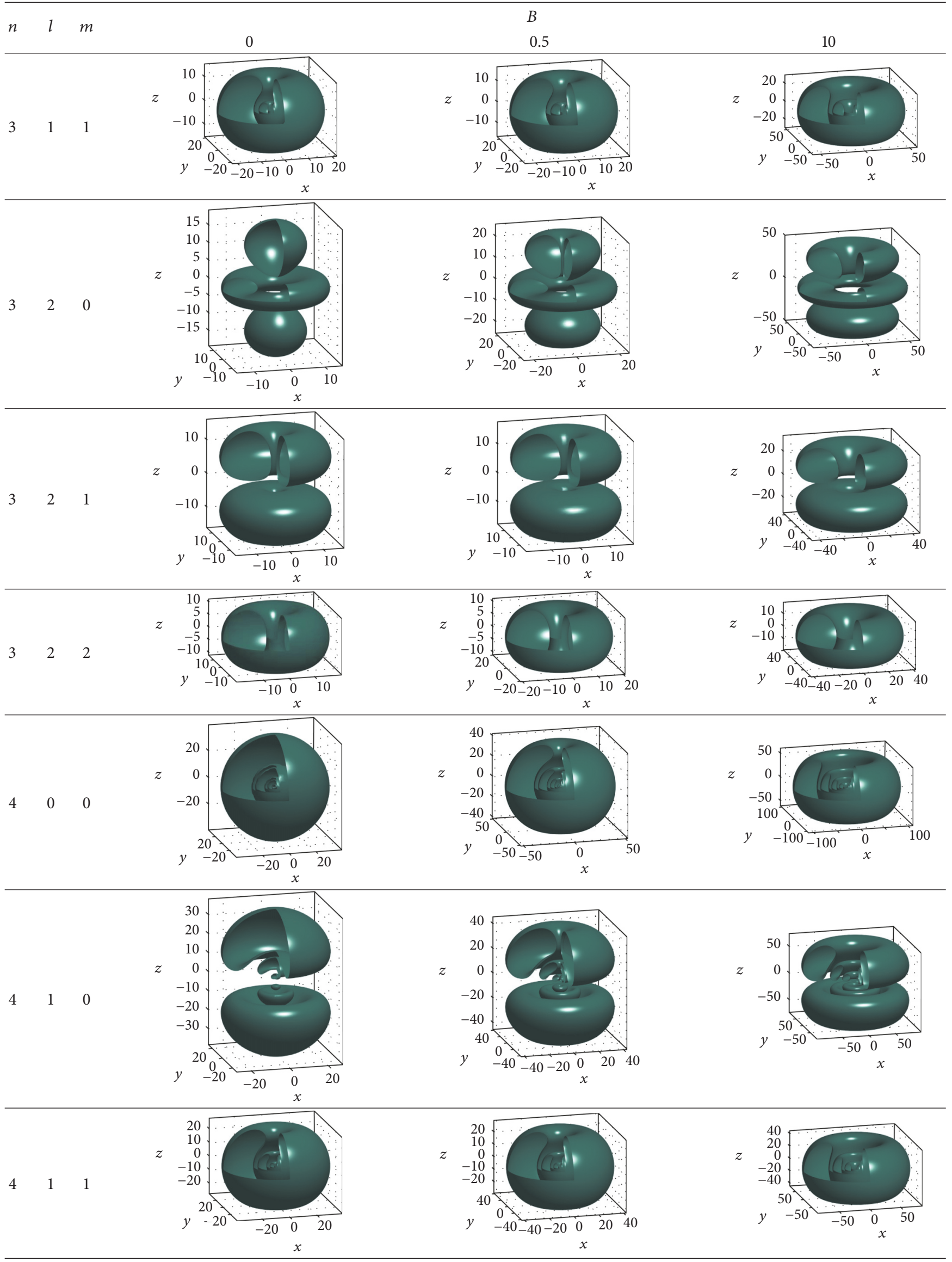


TABLe 2: Continued.

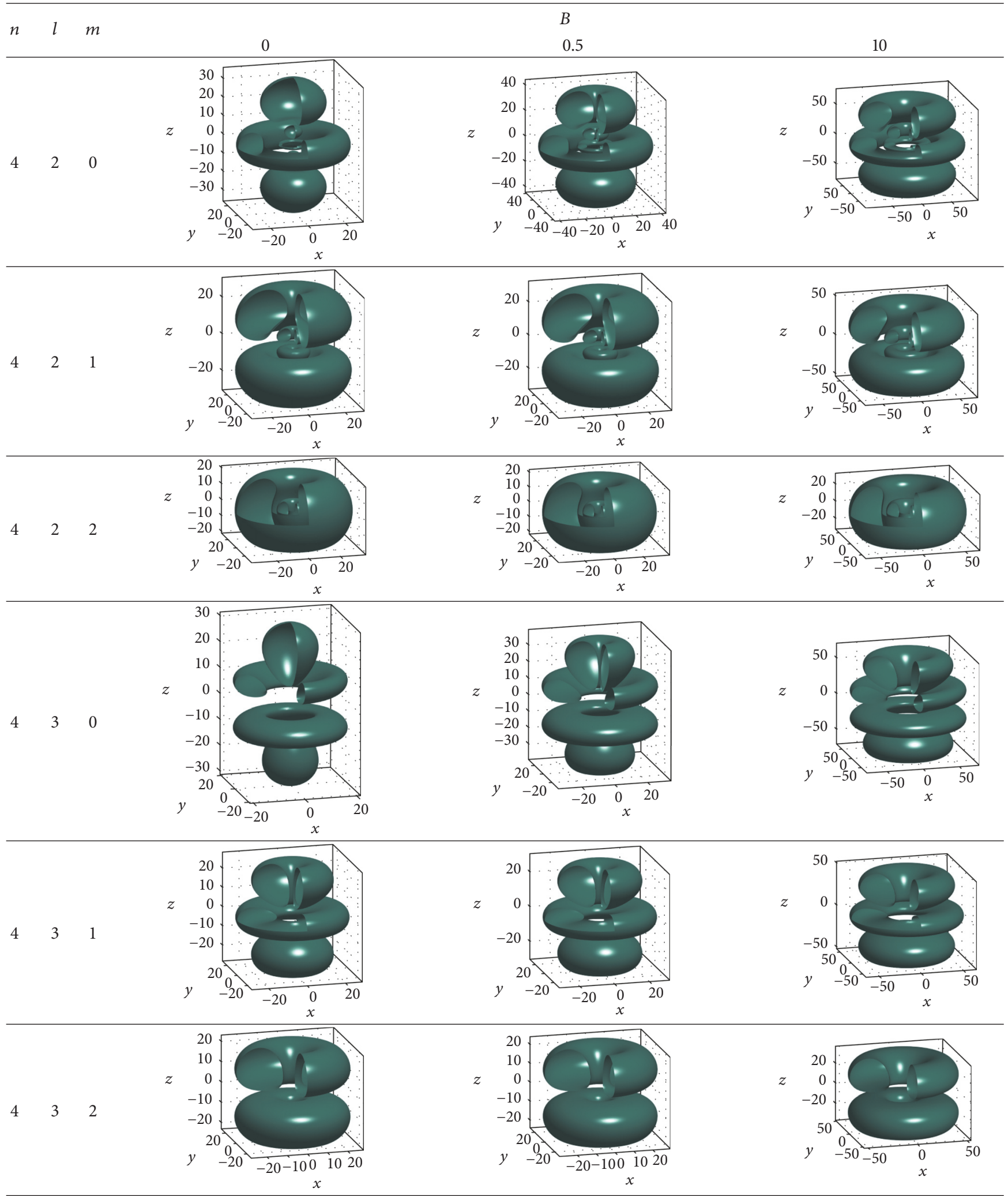


TABLe 2: Continued.

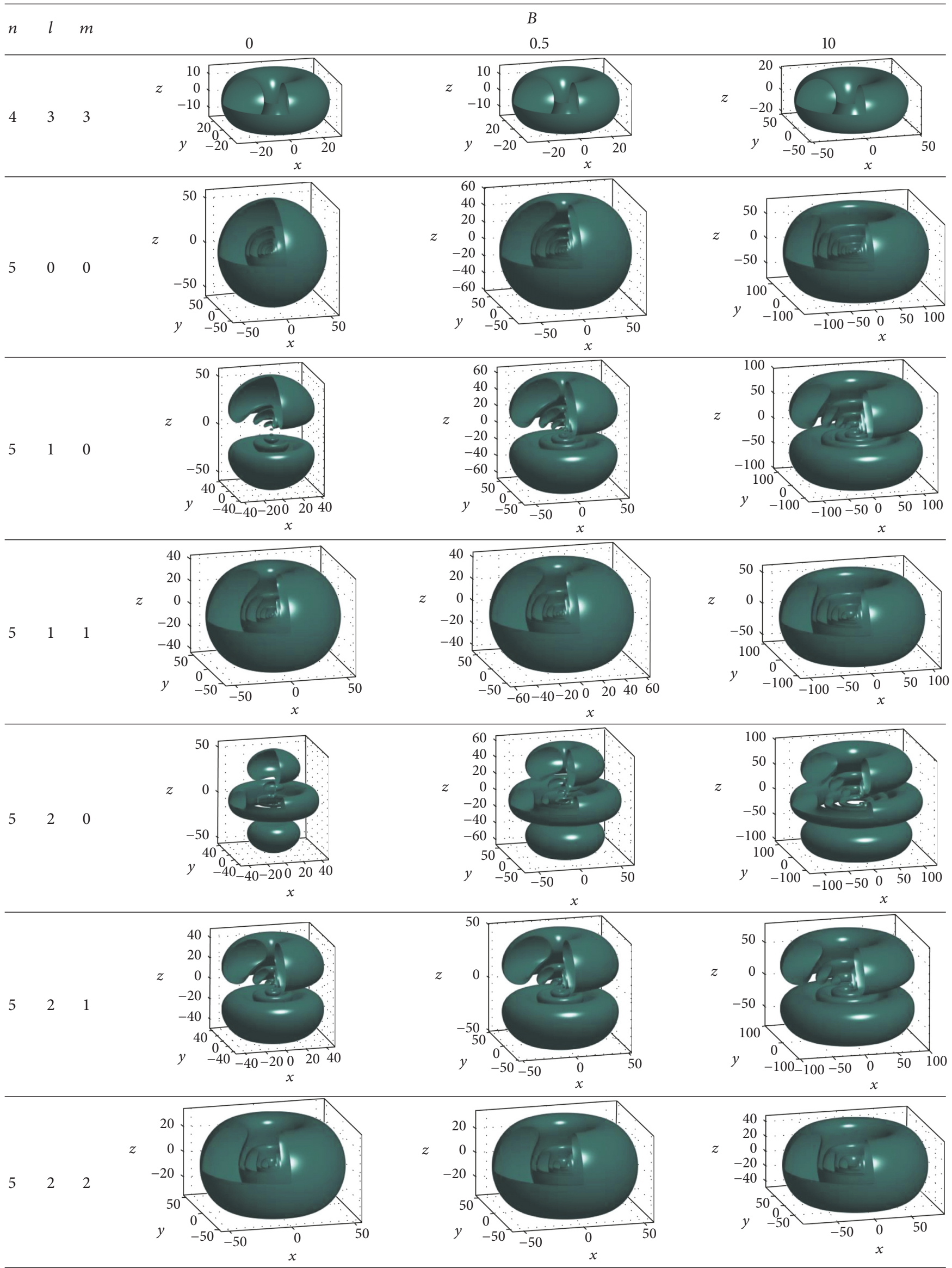


TABle 2: Continued.

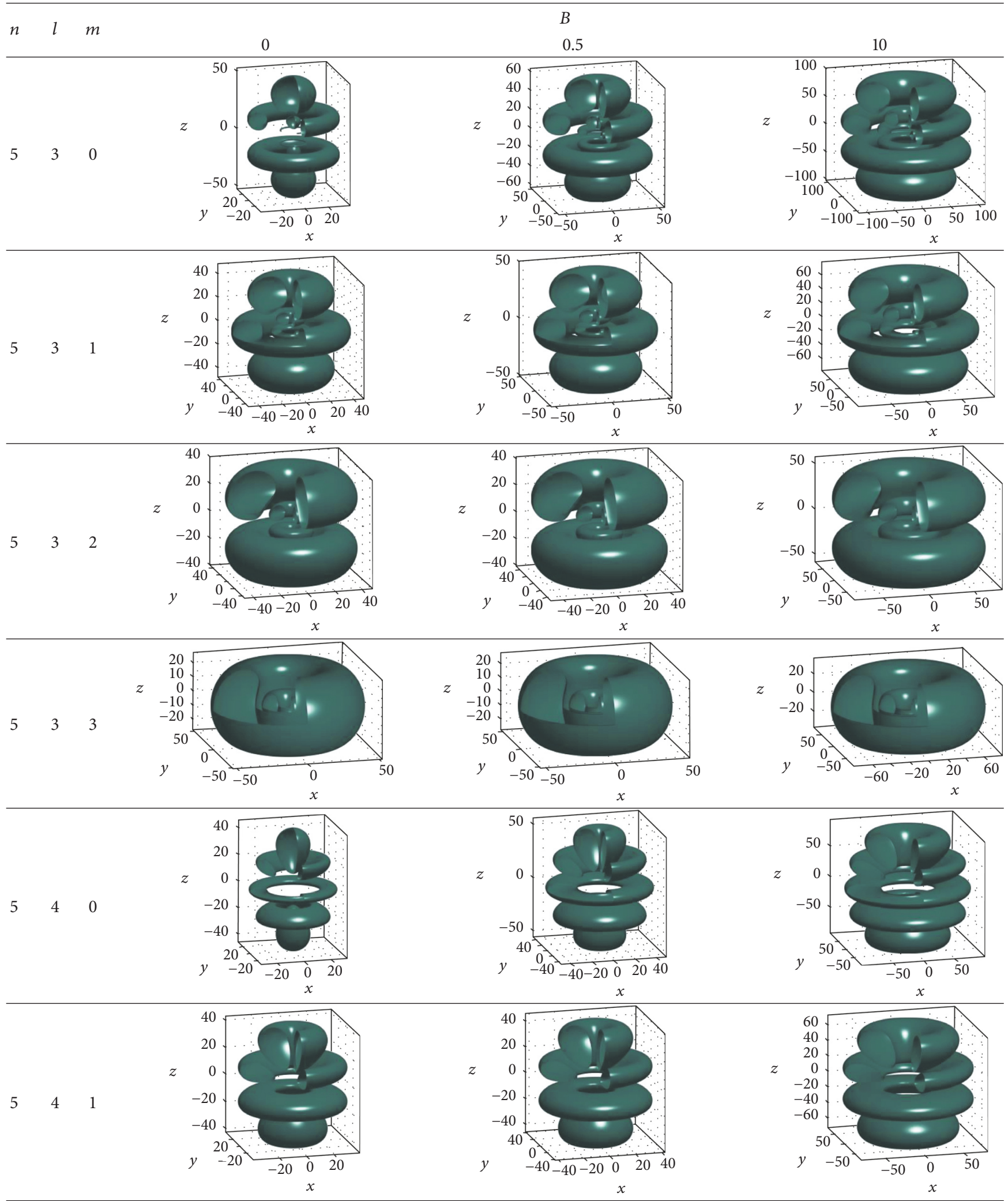


TABLE 2: Continued.

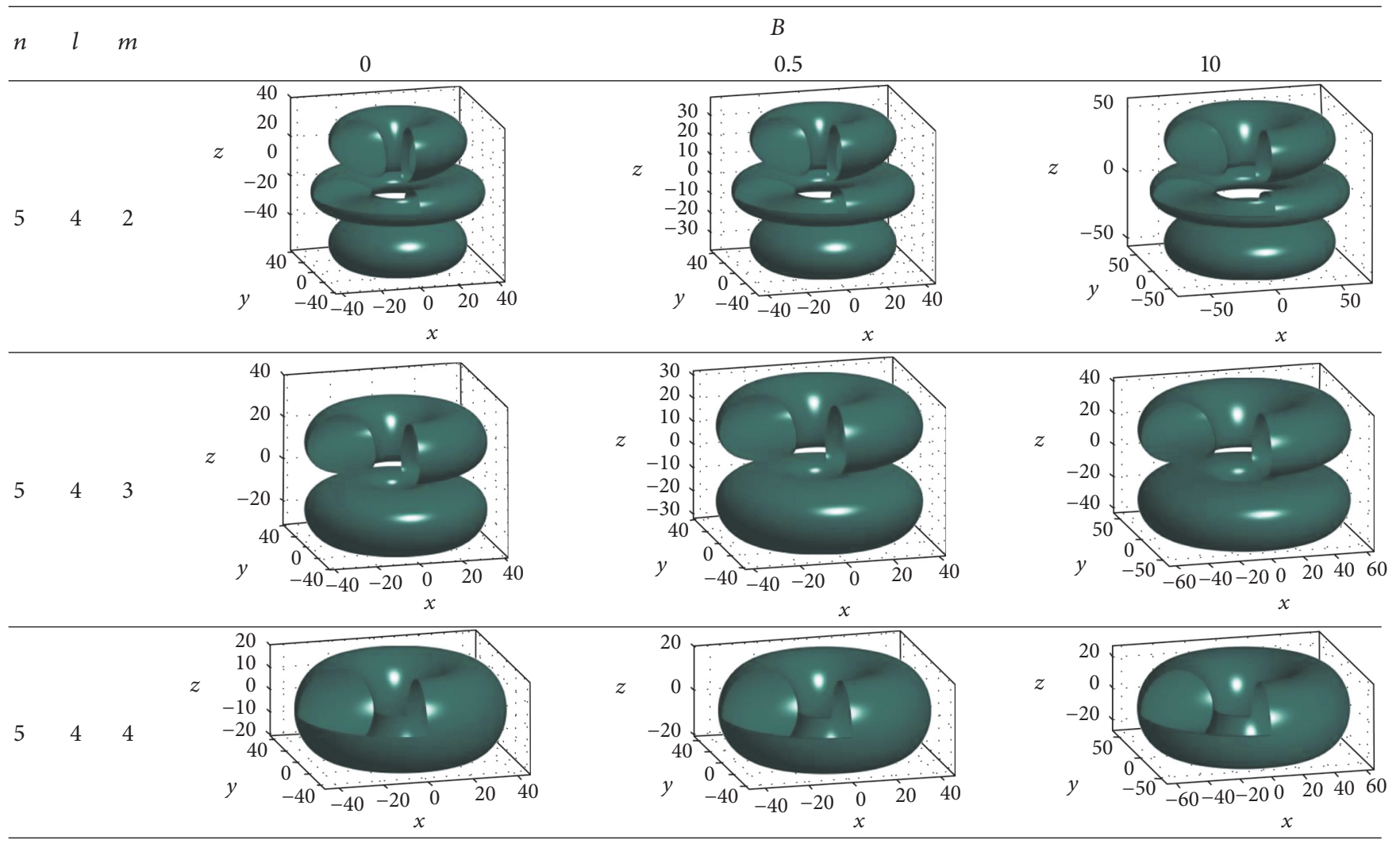

\section{Isosurface and Contour Visualizations of Space Probability Distributions}

It is well known that the space probability distributions for a moving particle at the position $\vec{r}=(r, \theta, \varphi)$ can be calculated by

$$
\rho=\left|\Psi_{n^{\prime} l^{\prime} m^{\prime}}(\vec{r})\right|^{2}=\frac{1}{2 \pi} \frac{u_{n^{\prime} l^{\prime}}^{2}(r)}{r^{2}} H_{l^{\prime} m^{\prime}}^{2}(\cos \theta) .
$$

Obviously, this formula is independent of the azimuth angle $\varphi$ and thus symmetric with respect to the $z$-axis. In order to display the space probability distribution, we will transform (15) from original spherical coordinates to the popular Cartesian coordinates through the coordinate transformations $r=\sqrt{x^{2}+y^{2}+z^{2}}$ and $\cos \theta=z / r$ and then obtain the corresponding space probability distribution $\rho(x, y, z)$.

For a given and definite space, we take a series of discrete positions and calculate their respective probability distribution values to realize their numerical calculations. To improve the graphic resolution, we take $N$ concrete positions in the whole space $(x, y, z)$ and calculate the density block, say $\operatorname{den}(N, N, N)$, which is composed of all values $w_{n^{\prime} l^{\prime} m^{\prime}}$ for all $N \times N \times N$ positions. In this work, we take $N=81$. For given quasi-quantum numbers $\left(n^{\prime}, l^{\prime}, m^{\prime}\right)$, we realize their isosurface (three-dimensional) and contour (two-dimensional) visualizations of the space probability distributions for different states $(n \leq 5)$ by using MATLAB program (see Tables 2 and 3).

\section{Discussions}

4.1. Variation of Space Probability Distribution with respect to the Numbers of Radial Nodes. In Table 2, we display the space probability distributions for three different cases: $b=0$ corresponding to the Coulomb potential and $b=0.5$ and $b=10$ corresponding to ring-shaped potentials. The unit in axis is taken as the Bohr radial $a_{0}$. To clearly visualize the internal structure of the graphics, we generate a section plane without considering those numerical values in the regions $x<0, y<0$, and $z>0$.

It is found that the graphics become compressed; that is, the space probability distributions elongate along with the $x$ - and $y$-axis and also the hole formed in the ring-shaped potentials expands towards the outside as the ring-shaped potential parameter $b$ increases. This can be well understood by the relations given in (7). We know that the value of the quasi-quantum number $m^{\prime}$ increases relatively for a given quantum number $m$. When $l=m$, the isosurface of the density distribution is spherical, but for the case $l \neq m$, its isosurface is circularly ring-shaped.

In Table 3, the space probability distribution is projected to plane yoz and is shown to be symmetric with respect to the $y$ - and $z$-axis. Here, we only plot the graphics in the first quadrant through magnifying proportionally the space probability $\left|\Psi_{n^{\prime} l^{\prime} m^{\prime}}(\vec{r})\right|^{2}$ and making it the maximum value to be 100 , while the interval is taken as 10 . There exists a corresponding balance among the density distributions in axis directions $x, y$, and $z$ since the sum of density 
TABLE 3: Contour of the space probability distribution in two-dimensional plane yoz.

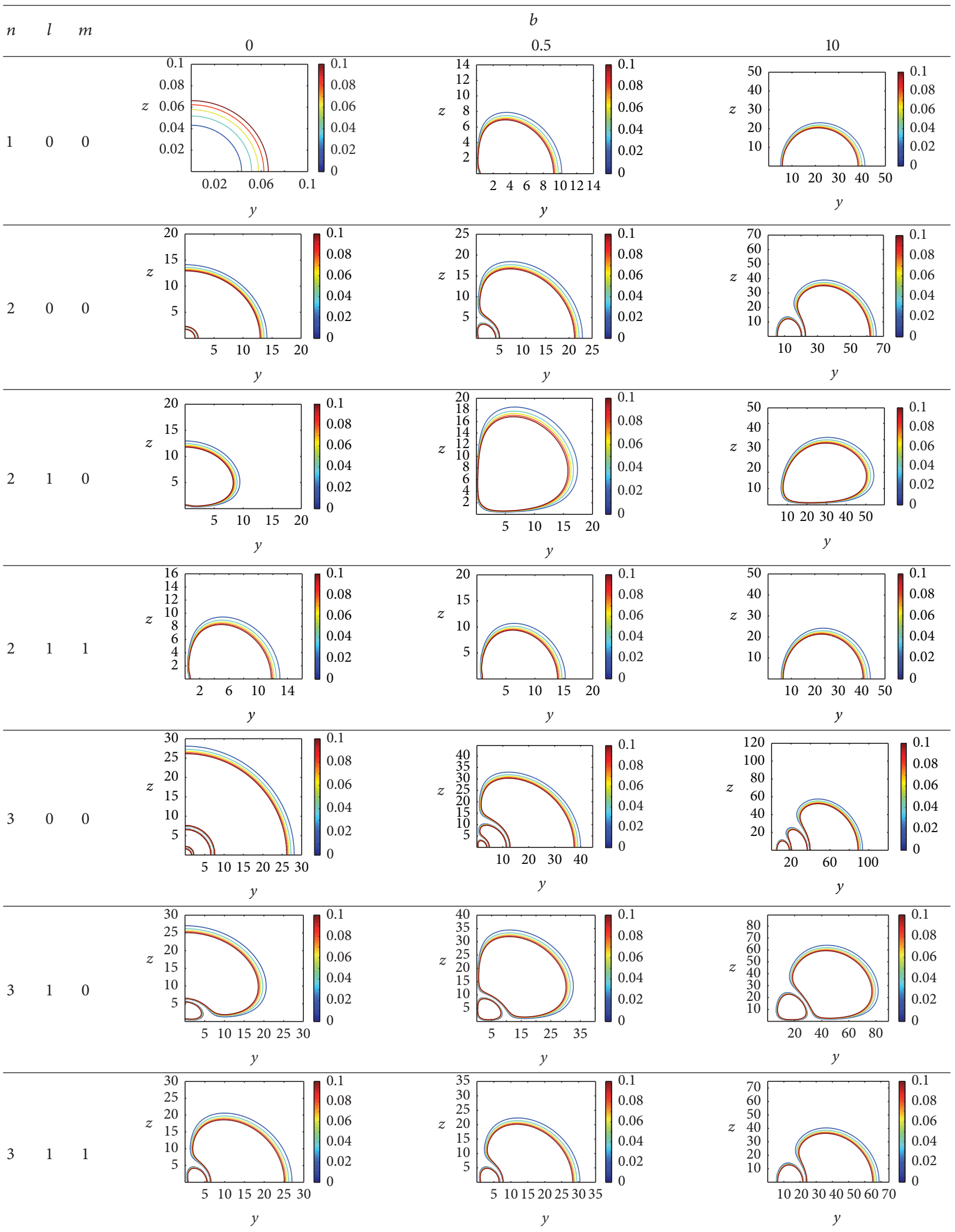


TABLE 3: Continued.

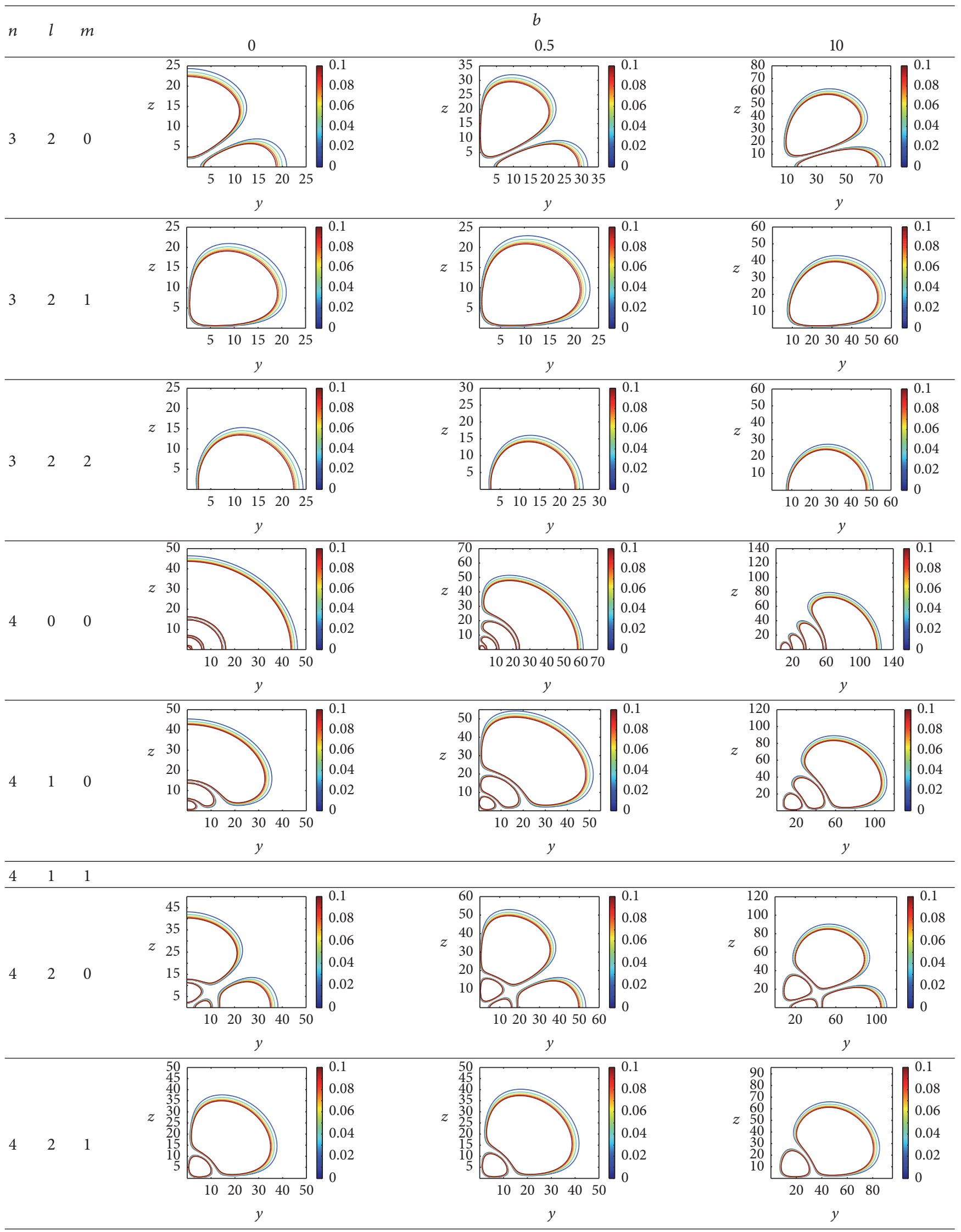


TABle 3: Continued.

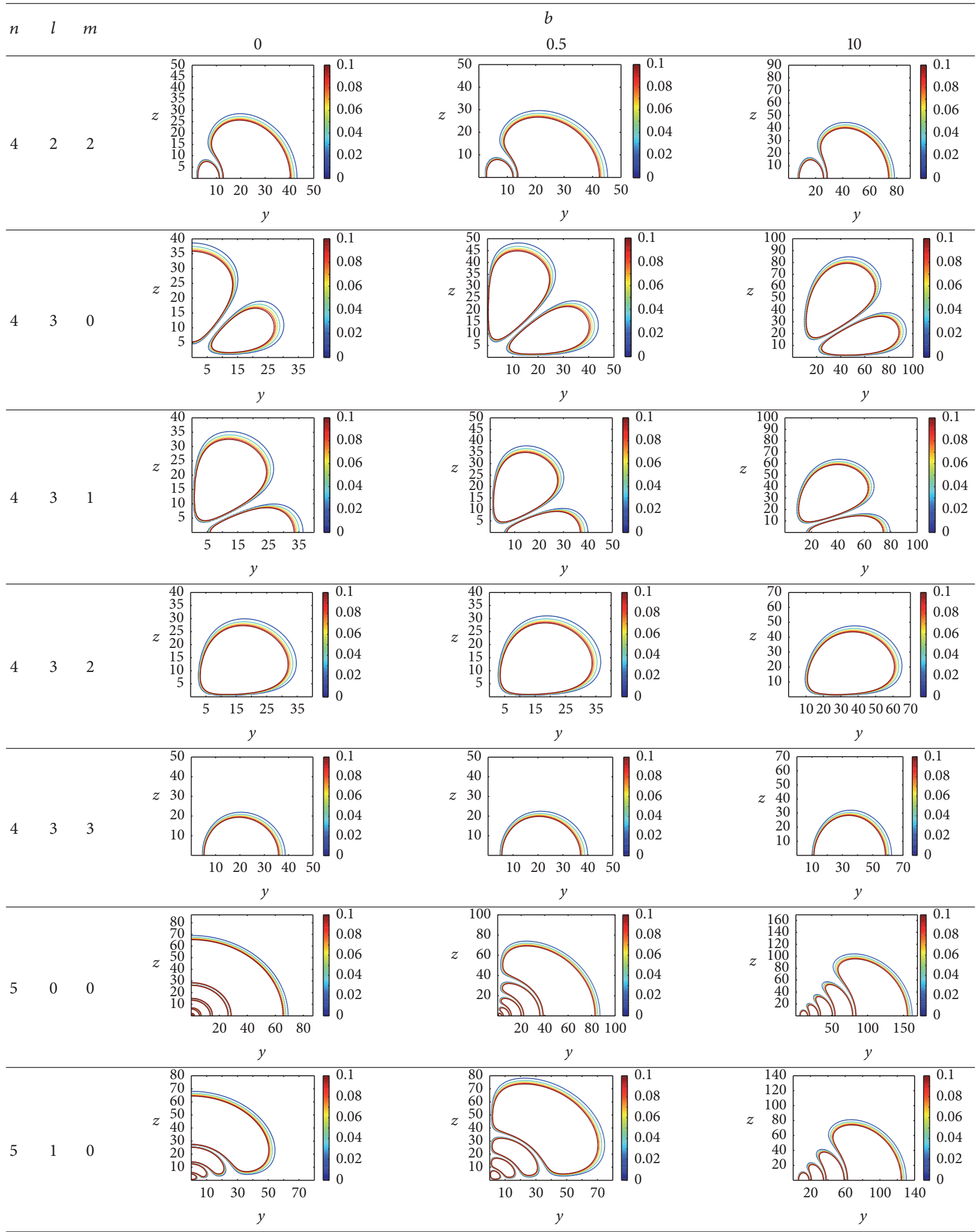


TABLe 3: Continued.

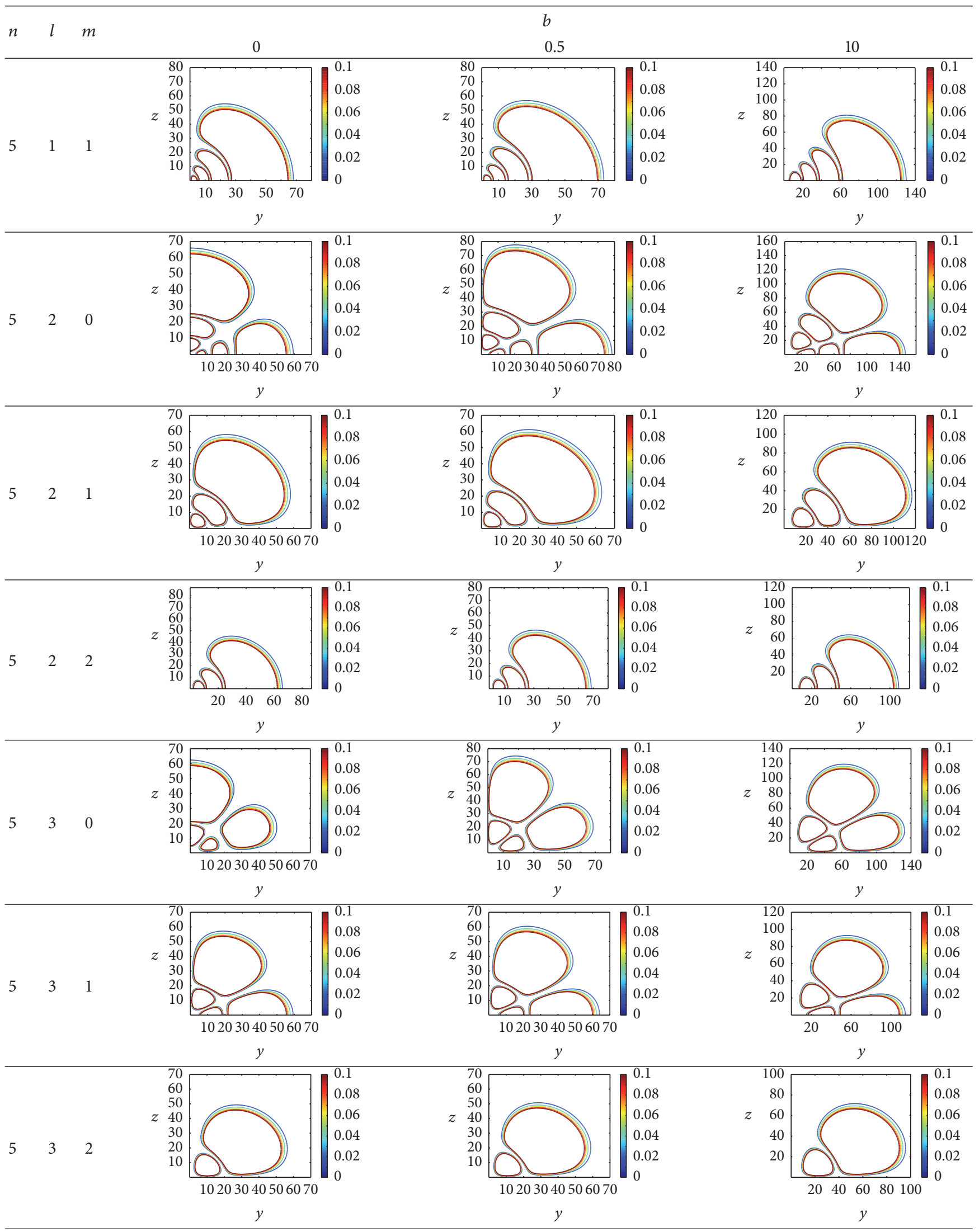


TABle 3: Continued.

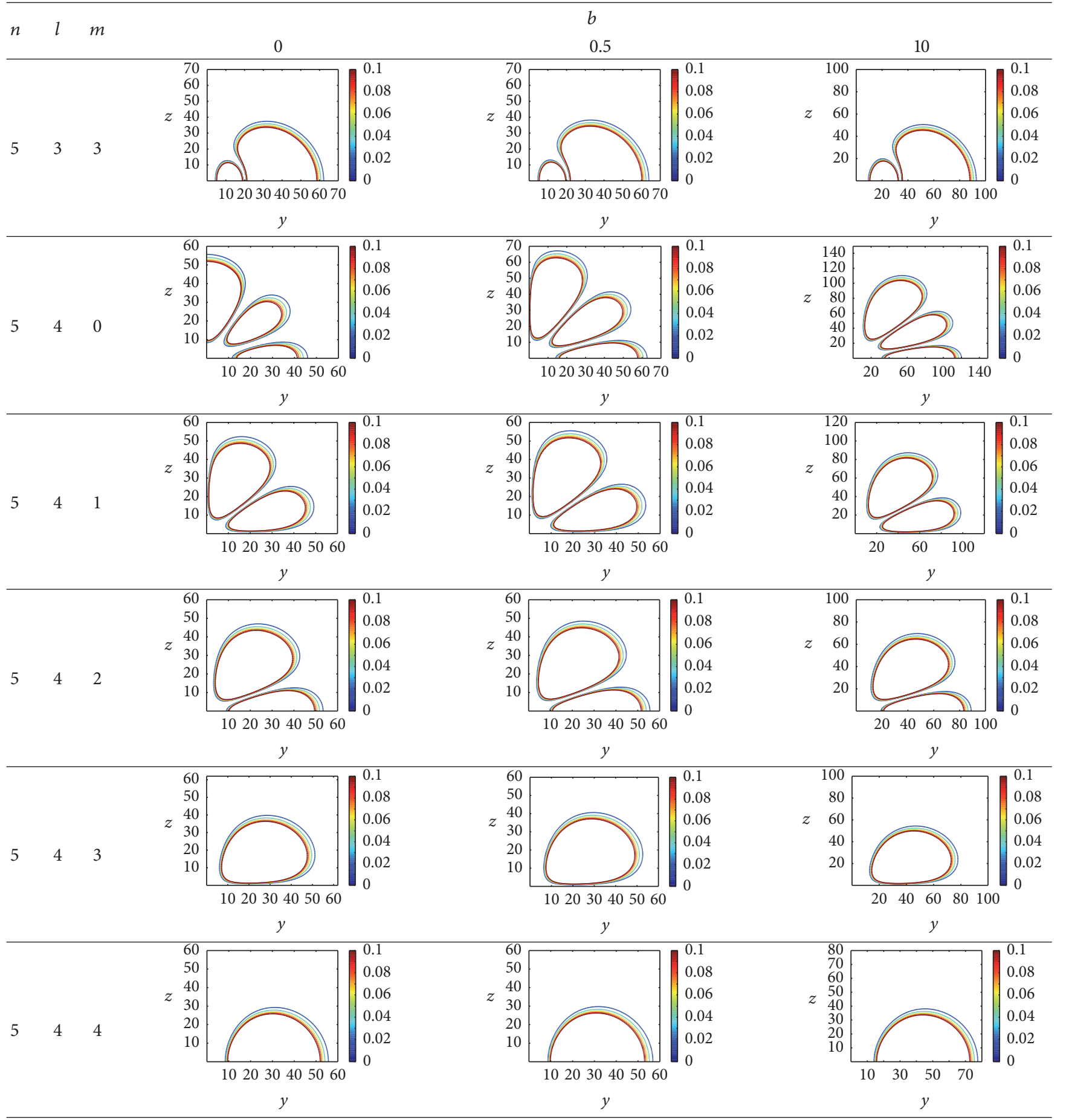

distributions is equal to unit according to the normalization condition.

4.2. Variation of the Space Probability Distributions with respect to Different Relative Probability Values $P$. In order to display the isosurface of the space probability distributions for different chosen relative probability values $P \in(0,100) \%$, we take the quantum numbers $(n, l, m)=(6,5,1)$ as a typical example in Table 4 . We find that, for smaller $P$, the particle is distributed to almost all position spaces, but for larger $P$ we notice that the particle moves towards the two poles of the $z$-axis.

4.3. Variations of the Space Probability Distribution with respect to Different Potential Parameters $b$. For fixed quantum numbers $m$ and $n_{\theta}$, it is known from (7) that the quasiquantum number $m^{\prime}$ becomes larger with increasing $b$ and thus leads to the increment of the quasi-quantum number 
TABLE 4: Space probability distributions for different chosen relative probability values $P$ for $(n, l, m)=(6,5,1)$.

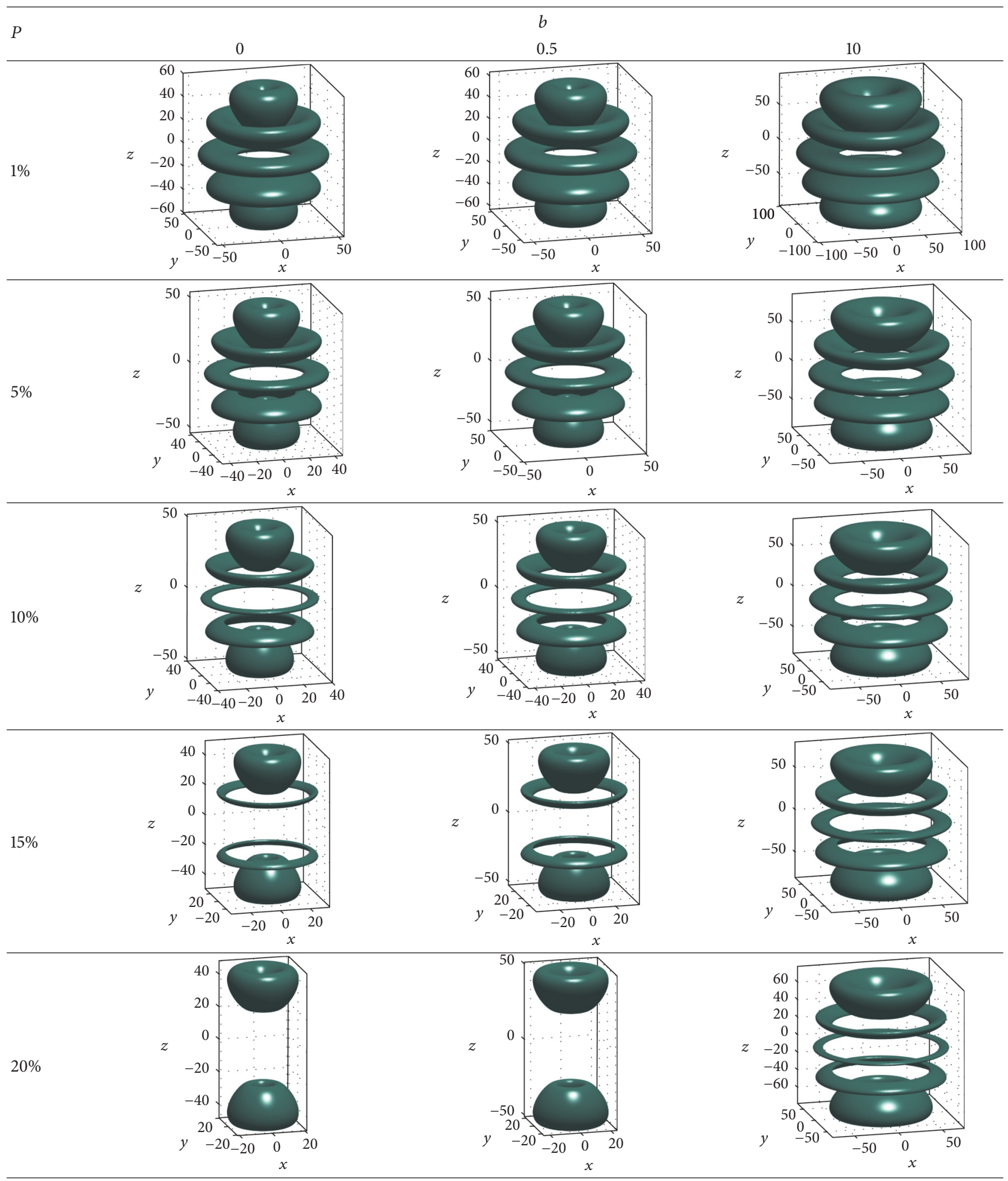


TABLE 4: Continued.

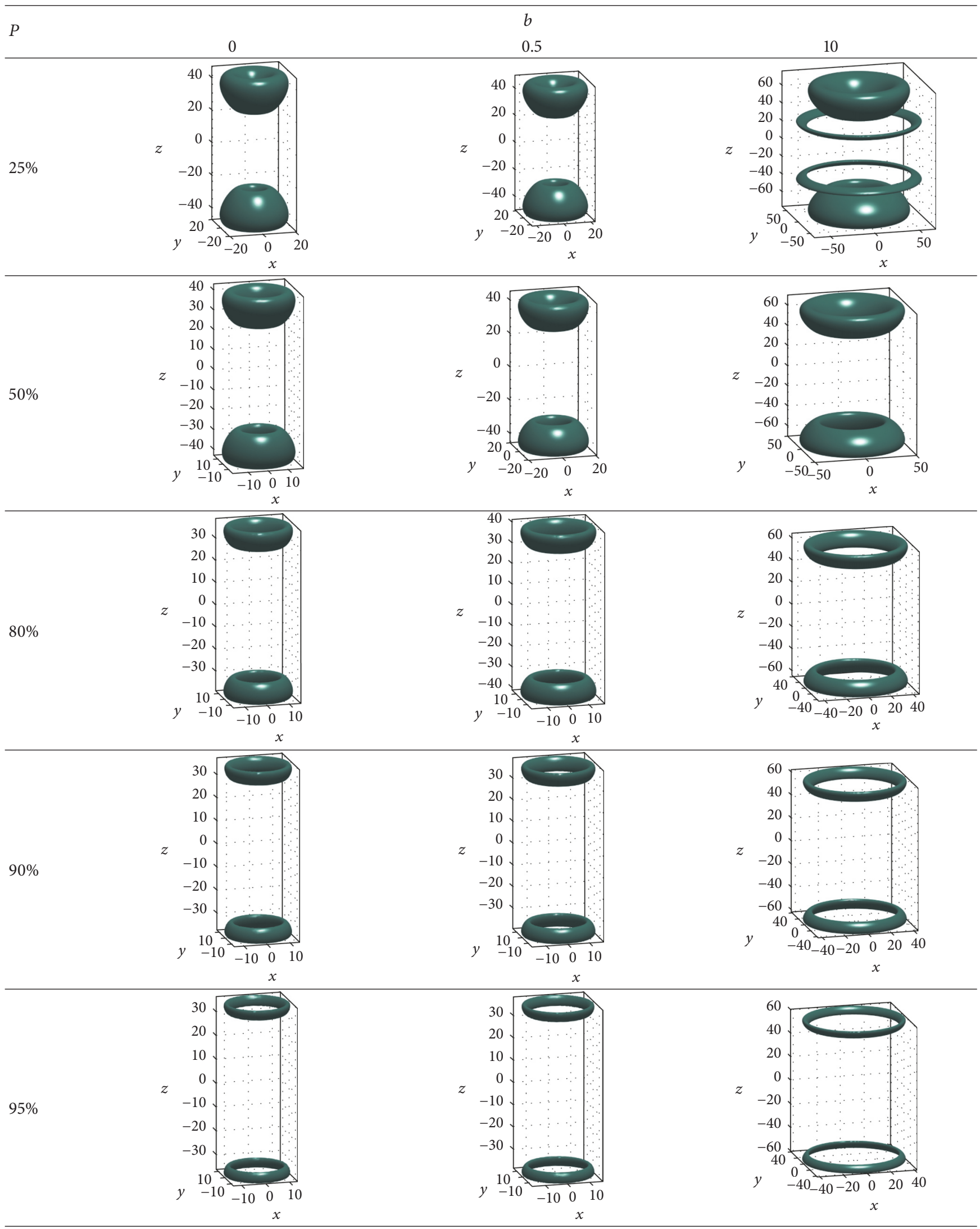


TABLE 5: Three-dimensional isosurface representation of $(4,0,0)$ for different positive values $b$.

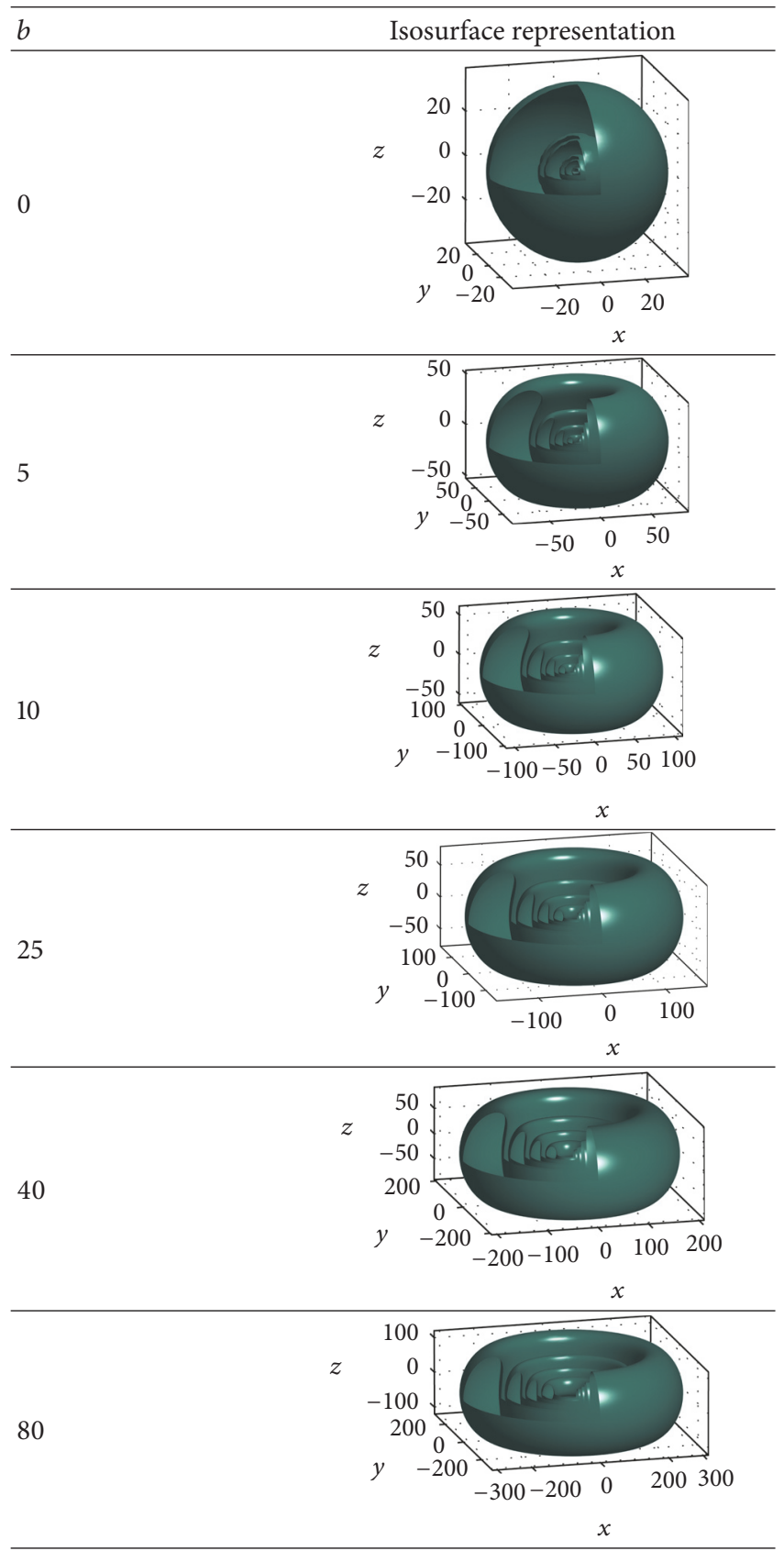

$l^{\prime}$. In Table 5, we plot the space probability distributions of the quantum state $(n, l, m)=(4,0,0)$ for the case $b>0$. Due to the change of quasi-quantum number $m^{\prime}$ which arises from the potential parameter $b$, the number of radial nodes is changed accordingly. This leads to the extensions of the space probability distributions along with the $x$-and $y$-axis when the parameter $b$ increases.

In Table 6, we give the comparison between the special cases for $b<0$ and $b>0$. Obviously, we find that the space probability distributions for the negative $b=-0.5$ in comparison with the special case $b=0$ are shrunk towards the origin of the graphics. On the contrary, those for the

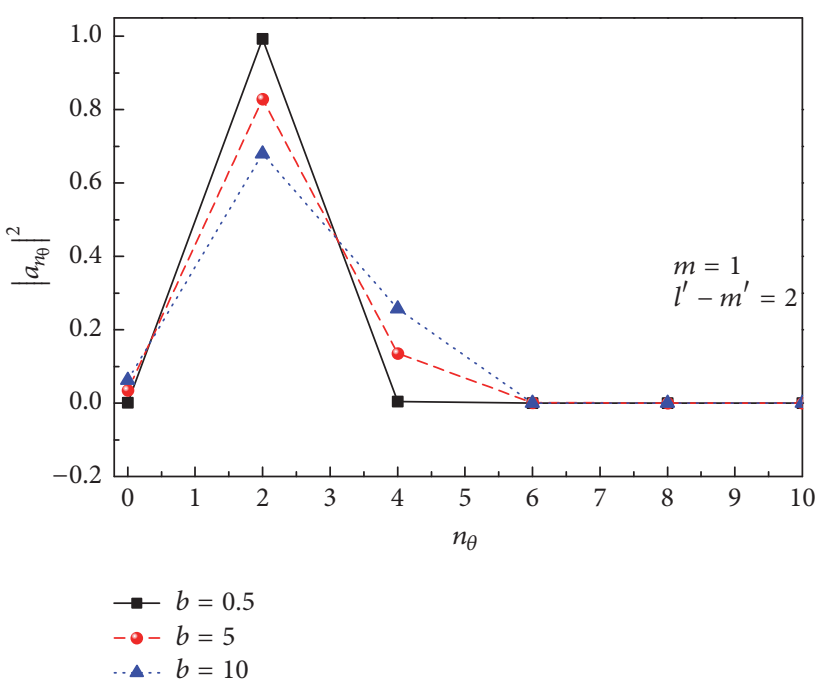

FIGURE 1: Relation between $n_{\theta}$ and $\left|a_{n_{\theta}}\right|^{2}$ for different values $b$.

positive case $b=0.5$ are enlarged towards the outside. This kind of phenomenon can be understood well from analyzing the contributions of the potential parameter $b$ to the Coulomb part. The original attractive Coulomb potential becoming bigger or smaller relatively depends on the choice of the negative or positive $b$. As a result, the attractive force acting on the moving particle becomes larger or smaller. Naturally, this causes the space probability distributions to be shrunk or extended.

\section{Expansion Coefficients of the Deformed Spherical Harmonics}

Since the spherical harmonics (9) are orthogonal and complete, then the deformed spherical harmonics (10) can be expanded by the usual spherical harmonics; that is,

$$
Y_{l^{\prime} m^{\prime}}(\theta, \varphi)=\sum_{l, m} a_{l m} Y_{l m}(\theta, \varphi)
$$

where the expansion coefficients can be calculated by

$$
\begin{aligned}
& a_{l m} \\
& =\sqrt{\frac{\left(2 l^{\prime}+1\right)(2 l+1)(l-m) ! \Gamma\left(l^{\prime}-m^{\prime}+1\right)}{4(l+m) ! \Gamma\left(l^{\prime}+m^{\prime}+1\right)}} \int_{-1}^{+1} P_{l}^{m}(x) \\
& \quad \cdot P_{l^{\prime}}^{m^{\prime}}(x) d x .
\end{aligned}
$$

In Figure 1, we plot the variation of the expansion coefficients with different parameters $b$ where we take $m=1, l^{\prime}-m^{\prime}=2$, and $b=0.5,5,10$. For $m \neq 1$, according to the orthogonal normalization condition in the $\varphi$ direction, each value of $a_{l m}$ is zero. For $m=1$, each value of $a_{l m}$ is also equal to zero for the case of odd value $l-m$. Thus, in Figure 1, we only present the case of even value $l-m$ for $m=1$. Notice that $P_{3}^{1}(x)$ is always the biggest, but the principal component $P_{3}^{1}(x)$ 
TABLE 6: Space probability distributions for positive and negative parameter $b$ for $(5,2,1)$.

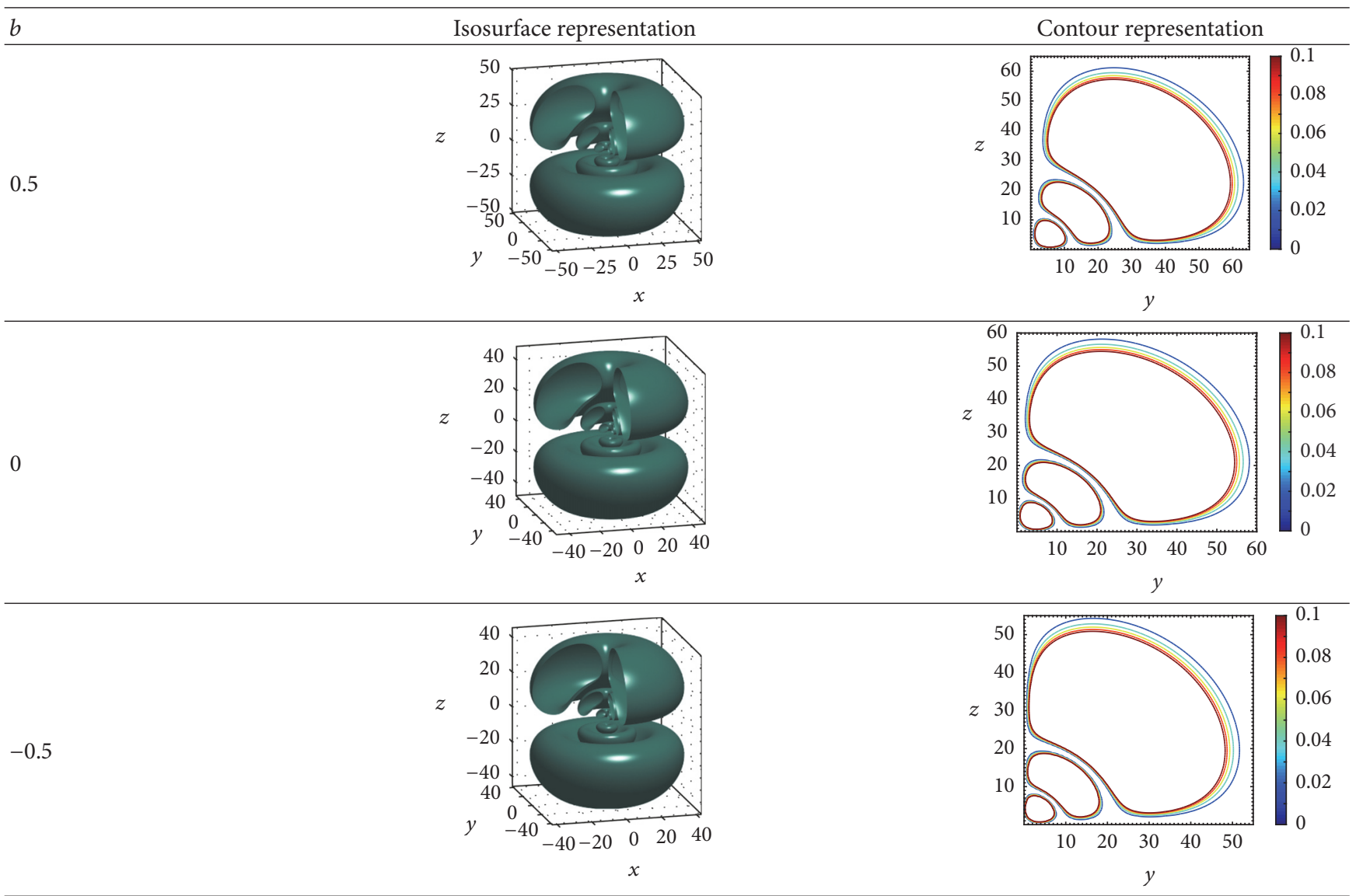

decreases gradually, and other components will increase as the parameter $b$ increases.

\section{Concluding Remarks}

In this work, we first presented the exact solutions to the single ring-shaped Coulomb potential and then realized the visualization of the space probability distributions for a moving particle within the framework of this potential. We have illustrated the two-dimensional (contour) and threedimensional (isosurface) visualizations for some given quasiquantum numbers $\left(n^{\prime}, l^{\prime}, m^{\prime}\right)$ by taking different ring-shaped potential parameters $b$. We have found that the space probability distributions of the moving particle in the cases of $l=m$ and $l \neq m$ are spherical and circularly ring-shaped, respectively. Moreover, we have studied the features of the relative values $P$ of the space probability distributions. As an illustration, we have discussed the special case, that is, the quantum numbers $(n, l, m)=(6,5,1)$, and noticed that the space probability distributions for the moving particle will move towards the two poles of the $z$-axis as the relative values $P$ increase. We have also studied the series expansion of the deformed spherical harmonics by using the orthogonal and complete spherical harmonics and found that the principal component decreases gradually and other components will increase as the potential parameter $b$ increases.

\section{Conflicts of Interest}

The authors declare that there are no conflicts of interest regarding the publication of this paper.

\section{Acknowledgments}

This work is supported by the National Natural Science Foundation of China under Grant no. 11275165 and partially by 20170938-SIP-IPN, Mexico. Professor Yuan You also acknowledges Jiangsu Overseas Research \& Training Program for University Prominent Young \& Middle-Aged Teachers and Presidents for the support.

\section{References}

[1] C. Quesne, "A new ring-shaped potential and its dynamical invariance algebra," Journal of Physics A.: Mathematical and General, vol. 21, no. 14, pp. 3093-3101, 1988.

[2] A. S. Zhedanov, "Hidden symmetry algebra and overlap coefficients for two ring-shaped potentials," Journal of Physics A.: Mathematical and General, vol. 26, no. 18, pp. 4633-4641, 1993.

[3] C. Y. Chen and D. S. Sun, "Exact solutions of a ring-shaped oscillator," Acta Photonica Sinica, vol. 30, p. 104, 2001.

[4] D. S. Sun and C. Y. Chen, "General formulas and recurrence formulas for radial matrix elements of ring shaped oscillator," Acta Photonica Sinica, vol. 30, p. 539, 2001. 
[5] S.-H. Dong, G.-H. Sun, and M. Lozada-Cassou, "An algebraic approach to the ring-shaped non-spherical oscillator," Physics Letters. A, vol. 328, no. 4-5, pp. 299-305, 2004.

[6] J. Y. Guo, J. C. Han, and R. D. Wang, "Pseudospin symmetry and the relativistic ring-shaped non-spherical harmonic oscillator," Physics Letters A, vol. 353, no. 5, pp. 378-382, 2006.

[7] H. Hartmann, "The motion of a body in a ring-shaped potential," Theoretica Chimica Acta, vol. 24, p. 201, 1972.

[8] H. Hartmann and R. Schuck, "Spin-orbit coupling for the motion of a particle in a ring-shaped potential," International Journal of Quantum Chemistry, vol. 18, p. 125, 1980.

[9] B. P. Mandal, "Path integral solution of noncentral potential," International Journal of Modern Physics A, vol. 15, no. 8, pp. 1225-1234, 2000.

[10] G. G. Blado, "Supersymmetric treatment of a particle subjected to a ring-shaped potential," International Journal of Quantum Chemistry, vol. 58, no. 5, pp. 431-439, 1996.

[11] C. C. Gerry, "Dynamical group for a ring potential," Physics Letters A, vol. 118, no. 9, pp. 445-447, 1986.

[12] L. Chetouani, L. Guechi, and T. F. Hammann, "Algebraic treatment of a general noncentral potential," Journal of Mathematical Physics, vol. 33, no. 10, pp. 3410-3418, 1992.

[13] C. Y. Chen, F. L. Lu, D. S. Sun, and S. H. Dong, "Analytic solutions of the double ring-shaped Coulomb potential in quantum mechanics," Chinese Physical B, Article ID 100302, p. 22, 2013.

[14] C.-Y. Chen, D.-S. Sun, and C.-L. Liu, "The general calculation formulas and the recurrence relations of radial matrix elements for Hartmann potential," Physics Letters A, vol. 317, pp. 80-86, 2003.

[15] H. Sobhani and H. Hassanabadi, "Davydov-chaban Hamiltonian in presence of time-dependent potential," Physics Letters $B$, vol. 760, pp. 1-5, 2016.

[16] H. Hassanabadi, M. Kamali, Z. Molaee, and S. Zarrinkamar, "Duffin-Kemmer-Petiau equation under Hartmann ringshaped potential," Chinese Physics C, vol. 38, no. 3, Article ID 033102, 2014.

[17] M. Chabab, A. El Batoul, M. Oulne, H. Hassanabadi, and S. Zare, "Scattering states of the Schrödinger equation with a position-dependent-mass and a non-central potential," Journal of the Korean Physical Society, vol. 69, no. 11, p. 1619, 2016.

[18] S. Zarrinkamar, K. Jahankohan, and H. Hassanabadi, “The spinorbit interaction in minimal length quantum mechanics; The case of a $(2+1)^{-}$dimensional Dirac oscillator," Canadian Journal of Physics, vol. 93, no. 12, pp. 1638-1641, 2015.

[19] A. N. Ikot, H. P. Obong, I. O. Owate, M. C. Onyeaju, and H. Hassanabadi, "Scattering state of klein-gordon particles by qparameter hyperbolic poschl-teller potential," Advances in High Energy Physics, Article ID 632603, 2015.

[20] W. Li, C. Y. Chen, and S. H. Dong, "Ring-shaped potential and a class of relevant integrals involved universal associated Legendre polynomials with complicated arguments," Advances in High Energy Physics, Article ID 7374256, 2017.

[21] C. Y. Chen, F. L. Lu, D. S. Sun, Y. You, and S. H. Dong, "Exact solutions to a class of differential equation and some new mathematical properties for the universal associated-Legendre polynomials," Applied Mathematics Letters, vol. 40, p. 90, 2015.

[22] D. S. Sun, Y. You, F. L. Lu, C. Y. Chen, and S. H. Dong, "The quantum characteristics of a class of complicated double ringshaped non-central potential," Physica Scripta, vol. 89, Article ID 045002, 2014.
[23] R. Sari A, A. Suparmi, and C. Cari, "Exact solution of Dirac equation for Scarf potential with new tensor coupling potential for spin and pseudospin symmetries using Romanovski polynomials," Chinese Physics B, vol. 25, Article ID 010301, 2016.

[24] I. S. Gradshteyn and I. M. Ryzhik, Table of Integrals, Series, and Products, Academic Press, Oxford, NY, USA, 6th edition, 2000.

[25] L. C. Andrews, Special Functions of Mathematics for Engineers, Oxford University Press, Oxford, NY, USA, 2nd edition, 1998.

[26] E. U. Condon and G. H. Shortley, The Theory of Atomic Spectra, Cambridge University press: Macmillan Company, NY, USA, 1935.

[27] P. Strange, Relativistic Quantum Mechanics, Oxford University Press, Oxford, NY, USA, 1998.

[28] L. D. Landau and E. M. Lifshitz, Quantum Mechanics (NonRelativistic Theory, Pergamon Press, Oxford, NY, USA, 3rd edition, 1977. 

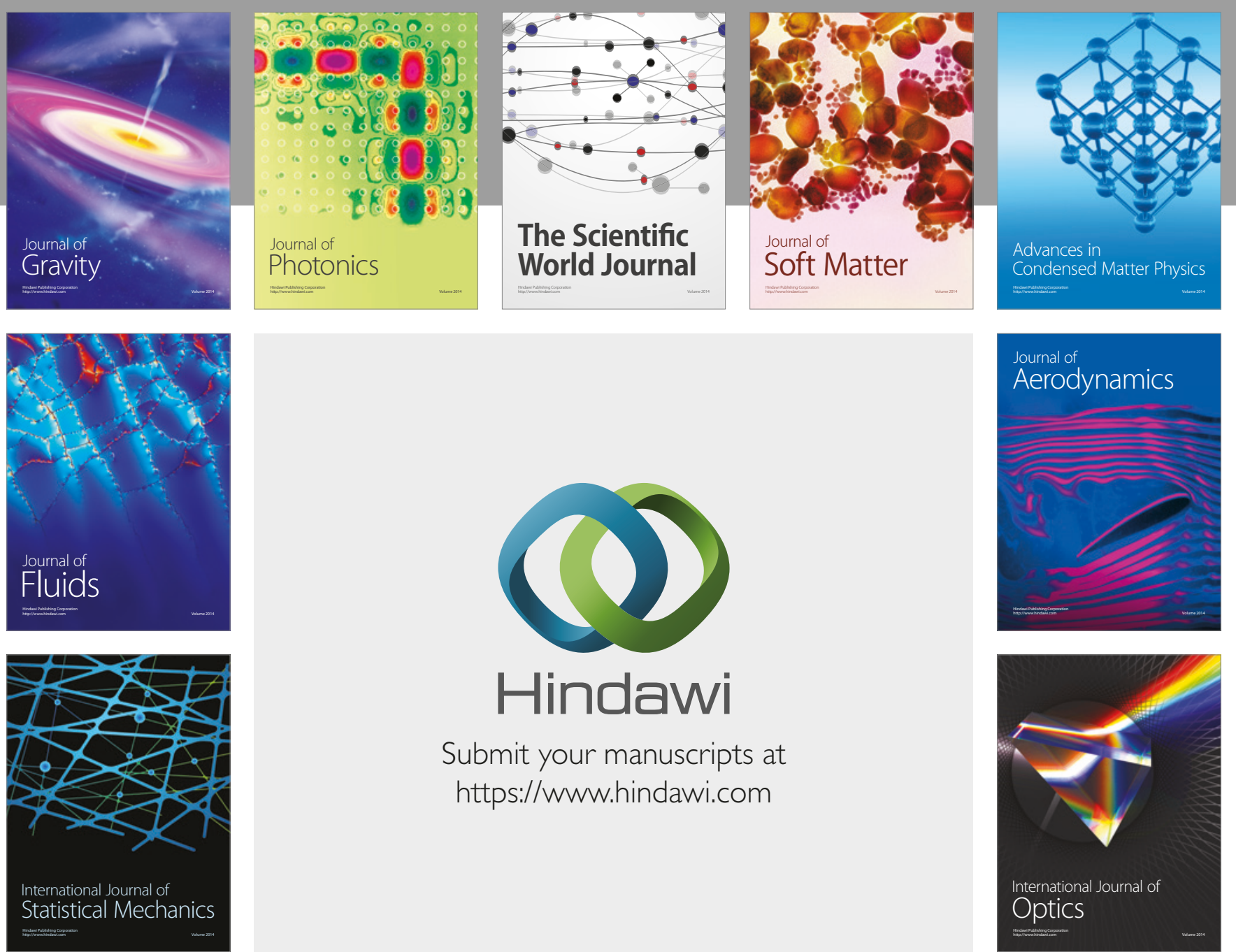

Submit your manuscripts at

https://www.hindawi.com
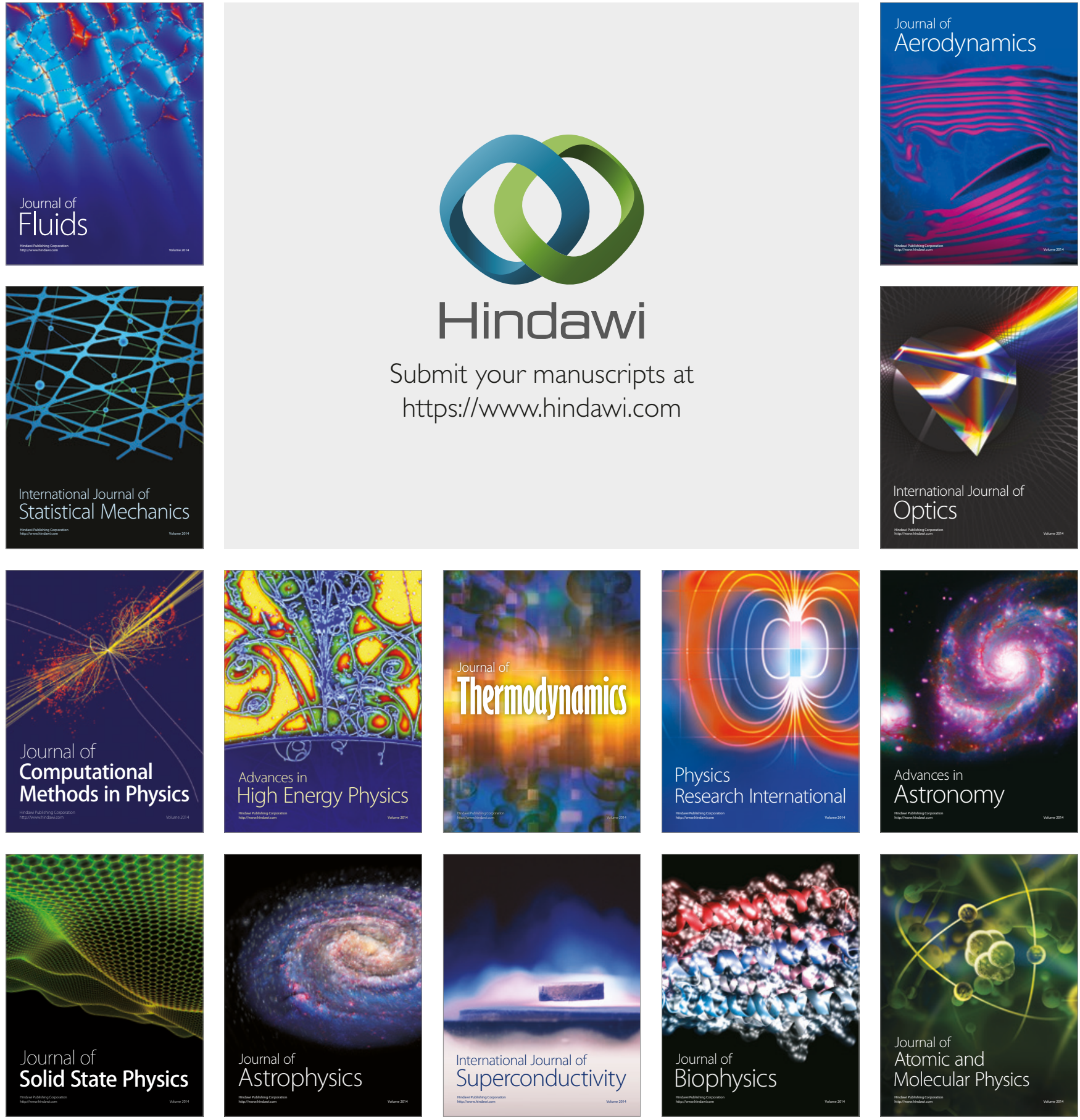